\title{
Star patterns on lake ice
}

\author{
Victor C. Tsai ${ }^{1, *}$ and J. S. Wettlaufer ${ }^{2, \dagger}$ \\ ${ }^{1}$ Department of Earth \& Planetary Sciences, Harvard University, Cambridge, Massachusetts 02138, USA \\ ${ }^{2}$ Department of Geology \& Geophysics and Department of Physics, Yale University, New Haven, Connecticut 06520-8109, USA
}

(Received 14 February 2007; published 18 June 2007)

\begin{abstract}
Star patterns, reminiscent of a wide range of diffusively controlled growth forms from snowflakes to Saffman-Taylor fingers, are ubiquitous features of ice-covered lakes. Despite the commonality and beauty of these "lake stars," the underlying physical processes that produce them have not been explained in a coherent theoretical framework. Here we describe a simple mathematical model that captures the principal features of lake-star formation; radial fingers of (relatively warm) water-rich regions grow from a central source and evolve through a competition between thermal and porous media flow effects in a saturated snow layer covering the lake. The number of star arms emerges from a stability analysis of this competition and the qualitative features of this meter-scale natural phenomenon are captured in laboratory experiments.
\end{abstract}

DOI: 10.1103/PhysRevE.75.066105

PACS number(s): 82.40.Ck, 45.70.Qj, 92.40.Vq

\section{INTRODUCTION}

The scientific study of the problems of growth and form occupies an anomalously broad set of disciplines. Whether the emergent patterns are physical or biological in origin, their quantitative description presents many challenging and compelling issues in, for example, applied mathematics [1], biophysics [2], condensed matter [3], and geophysics [4], wherein the motion of free boundaries is of central interest. In all such settings a principal goal is to predict the evolution of a boundary that is often under the influence of an instability. Here we study a variant of such a situation that occurs naturally on the frozen surfaces of lakes.

Lakes commonly freeze during a snowfall. When a hole forms in the ice cover, relatively warm lake water will flow through it and hence through the snow layer. In the process of flowing through and melting the snow, this warm water creates dark regions. The pattern so produced looks starlike (see Fig. 1), and we refer to it as a "lake star." These compelling features have been described qualitatively a number of times (e.g., [5-7]), but work on the formation process itself has been solely heuristic. Knight [5] outlines a number of the physical ideas relevant to the process, but does not translate them into a predictive framework to model field observations. Knight's main idea is that locations with faster flow rates melt preferentially, leading to even faster flow rates and therefore to an instability that results in fingers. This idea has features that resemble those of many other instabilities such as, for example, those observed during the growth of binary alloys [8], in the flow of water through a rigid hot porous medium [9], or in more complex geomorphological settings [10], and we structure our model accordingly.

Katsaros [6] and Woodcock [7] attribute the holes from which the stars emanate and the patterns themselves to thermal convection patterns within the lake, but do not measure or calculate their nature. However, often the holes do not

\footnotetext{
*Electronic address: vtsai@fas.harvard.edu

${ }^{\dagger}$ Electronic address: john.wettlaufer@yale.edu
}

exhibit a characteristic distance between them but rather form from protrusions (e.g., sticks that poke through the ice surface) [5] and stars follow, thereby ruling out a convective mechanism as being necessary to explain the phenomena. The paucity of literature on this topic provides little more than speculation regarding the puncturing mechanism but lake stars are observed in all of these circumstances. Therefore, while hole formation is necessary for lake-star formation, its origin does not control the mechanism of pattern formation, which is the focus of the present work.

\section{THEORY}

The water level in the hole is higher than that in the wet snow-slush layer [5] and hence we treat this warm water [11] region as having a constant height above the ice, or equivalently a constant pressure head, which drives flow of water through the slush layer, which we treat as a Darcy flow of water at $0{ }^{\circ} \mathrm{C}$. We model the temperature field within the liquid region with an advection-diffusion equation and impose an appropriate (Stefan) condition for energy conservation at the water-slush interface. The water is everywhere incompressible. Finally, the model is closed with an outer boundary condition at which the pressure head is assumed known.

Although we lack in situ pressure measurements, circular water-saturated regions (a few meters in radius) are observed around the lake stars. Hence, we assume that the differential pressure head falls to zero somewhere in the vicinity of this circular boundary. The actual boundary at which the differential pressure head is zero is not likely to be completely uniform (as in Fig. 4 of Knight [5]), but treating it as uniform is a good approximation in the linear regime of our analysis. Finally, we treat the flow as two dimensional. Thus, although the water in direct contact with ice must be at $0{ }^{\circ} \mathrm{C}$, we consider the depth-averaged temperature, which is above freezing. Additionally, the decreasing pressure head in the radial direction must be accompanied by a corresponding drop in water level. Therefore, although the driving force is more accurately described as deriving from an axisymmetric gravity current, the front whose stability we assess is con- 


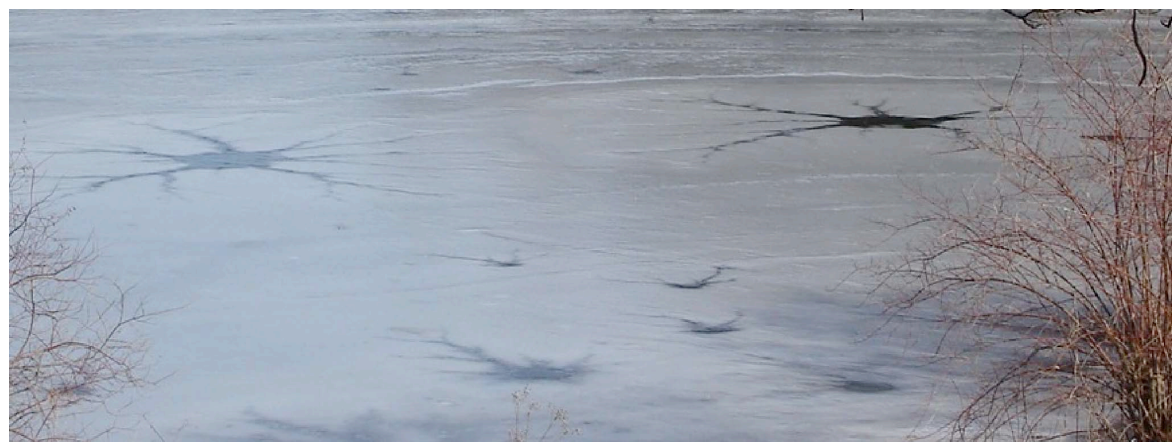

FIG. 1. (Color online) Typical lake-star patterns. The branched arms are approximately $1 \mathrm{~m}$ in length. Quonnipaug Lake, Guilford, Connecticut, 8 March 2006. trolled by the same essential physical processes that we model herein. Our analysis could be extended to account for these three-dimensional effects.

The system is characterized by the temperature $T$, a Darcy fluid velocity $\mathbf{u}$, pressure $p$, and an evolving liquid-slush interface $a$. The liquid properties are $\kappa$ (thermal diffusivity), $C_{P}$ (specific heat at constant pressure), and $\mu$ (dynamic viscosity) and the slush properties are $\Pi$ (permeability), $\xi$ (solid fraction), and $L$ (latent heat). We nondimensionalized the equations of motion by scaling the length, temperature, pressure, and velocity with $r_{0}, T_{0}, p_{0}$, and $\Pi p_{0} / \mu r_{0}$, respectively. Thus, our model consists of the following system of dimensionless equations:

$$
\begin{gathered}
\frac{\partial \theta}{\partial t}+\mathbf{u} \cdot \nabla \theta=\epsilon \nabla^{2} \theta, \quad r_{i}<r<a(\phi, t), \\
\theta=0, \quad a(\phi, t)<r<1, \\
p=1, \quad r_{i}<r<a(\phi, t), \\
\nabla^{2} p=0, \quad a(\phi, t)<r<1, \\
\nabla \cdot \mathbf{u}=0, \quad r_{i}<r<a(\phi, t), \\
\left.\mathbf{u}\right|_{a_{-}}=\left.\mathbf{u}\right|_{a_{+}}, \quad r=a(\phi, t), \\
\mathbf{u}=-\nabla p, \quad a(\phi, t)<r<1,
\end{gathered}
$$

with boundary conditions

$$
\begin{gathered}
\dot{a}=-\frac{\epsilon}{S} \nabla \theta, \quad r=a(\phi, t), \\
\theta= \begin{cases}1, & r=r_{i} \\
0, & r=a(\phi, t) \\
0, & r=1,\end{cases}
\end{gathered}
$$

and

$$
p= \begin{cases}1, & r=r_{i} \\ 1, & r=a(\phi, t) \\ 0, & r=1,\end{cases}
$$

where (1) describes the temperature evolution in the liquid, (4) and (5) describe mass conservation with a Darcy flow (7) in the slush, (8) is the Stefan condition, and (9) and (10) are the temperature and pressure boundary conditions, respectively (see Fig. 2). Note that (3) and (5) can both be satisfied since the liquid region has an effectively infinite permeability.

The dimensionless parameters $\epsilon$ and $S$ of the system are given by

$$
\epsilon \equiv \frac{\kappa}{u_{0} r_{0}} \quad \text { and } \quad S \equiv \frac{\xi L}{C_{P} T_{0}}
$$

which describe an inverse Péclet number and a Stefan number, respectively. Because the liquid must be less than or equal to $4^{\circ} \mathrm{C}$, we make the conservative estimates that $T_{0}$ $<4{ }^{\circ} \mathrm{C}, \xi>0.3$, and use the fact that $L / C_{P} \approx 80{ }^{\circ} \mathrm{C}$ from which we see that $S>6 \gg 1$. Using $\kappa \approx 10^{-7} \mathrm{~m}^{2} \mathrm{~s}^{-1}$ and the field observations of Knight [5] to constrain $u_{0}\left(1<u_{0}\right.$ $<10 \mathrm{~cm} / \mathrm{h})$ and $r_{0}\left(0.3<r_{0}<3 \mathrm{~m}\right)$, we find that $\epsilon<0.1 \ll 1$. We therefore employ the quasistationary $(S \gg 1)$ and large Péclet number $(\epsilon \ll 1)$ approximations, and hence Eqs. (1)-(10) are easily solved for a purely radial flow with cylindrical symmetry (no $\phi$ dependence) and circular liquidslush interface $a_{0}$. This (boundary layer) solution is

$$
\mathbf{u}=u \hat{r}=-\frac{1}{\ln \left(a_{0}\right)} \frac{1}{r} \hat{r}, \quad r_{i}<r<1,
$$

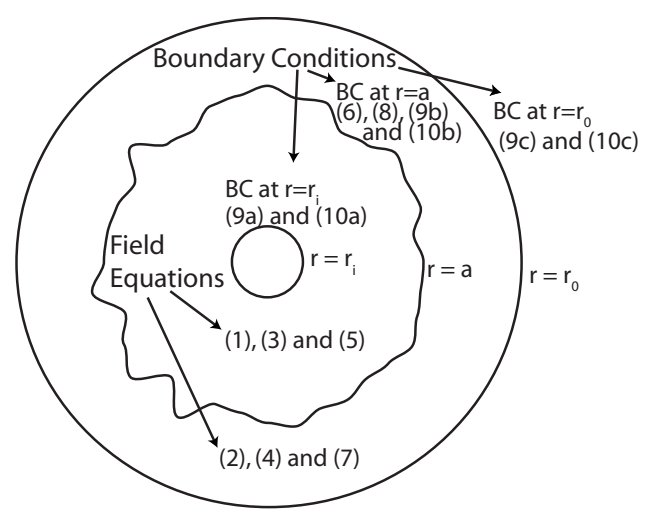

FIG. 2. Schematic of the geometry of the model. The perspective is looking down on a nascent star. The equations (refer to text for numbering) are shown in the domains of the system where they are applicable. 


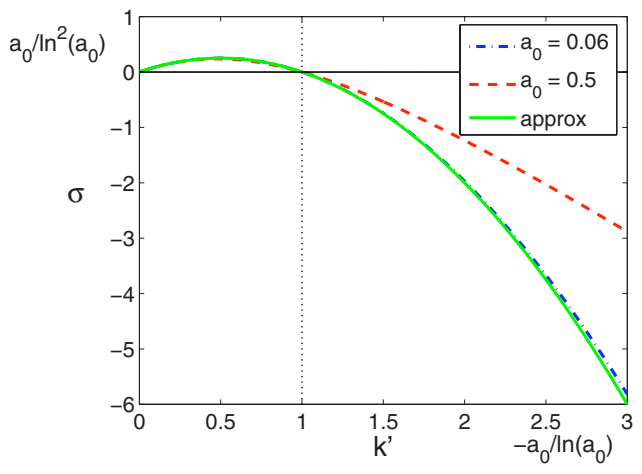

FIG. 3. (Color online) Stability curve: Nondimensional growth rate $\sigma$ versus nondimensional wave number $k^{\prime}$. Scales for the axes are given at the upper left ( $\sigma$ axis) and the lower right corners $\left(k^{\prime}\right.$ axis). $\sigma$ is plotted for the range of plausible $a_{0}$ [dot-dashed (blue) and dashed (red) curves] and for the approximation (18) [solid (green) curve].

$$
\begin{gathered}
p_{b}=\frac{\ln (r)}{\ln \left(a_{0}\right)}, \quad r>a_{0}, \\
\theta_{0}=1-\left(\frac{r}{a_{0}}\right)^{(1 / \epsilon)\left[-1 / \ln \left(a_{0}\right)+2 \epsilon\right]}, \quad r<a_{0}, \\
\frac{S a_{0} \dot{a}_{0}}{-1 / \ln \left(a_{0}\right)+2 \epsilon}=1,
\end{gathered}
$$

where Eq. (15) has an approximate implicit solution for $a_{0}$ given by

$$
\frac{a_{0}^{2}}{4}-\frac{1}{2} a_{0}^{2} \ln \left(a_{0}\right)=\frac{t}{S}
$$

We perform a linear stability analysis around this quasisteady cylindrically symmetrical flow. Proceeding in the usual way, we allow for scaled perturbations in $\theta$ and $a$ with scaled wave number $k^{\prime}=\epsilon k$, nondimensional growth rate $\sigma$, and amplitudes $f(r)$ and $g$, respectively. Keeping only terms linear in $\epsilon, 1 / S$, and $g$, we solve (4) subject to (10), substitute into (6), and satisfy (5) and (1). This gives the nondimensional growth rate $(\sigma)$ as a function of scaled wave number $\left(k^{\prime}\right)$ :

$$
\sigma=\frac{1}{2 a_{0} \ln ^{2}\left(a_{0}\right) S}\left[\sqrt{1+4 k^{\prime 2} \ln ^{2}\left(a_{0}\right)}-1\right]\left(\frac{a_{0}}{-k^{\prime} \ln \left(a_{0}\right)}-1\right) .
$$

Equation (17) can be approximated in $0 \leqslant x \leqq 1$ as

$$
\sigma \approx \frac{a_{0}}{\ln ^{2}\left(a_{0}\right) S} x(1-x),
$$

where $x \equiv-k^{\prime} \ln \left(a_{0}\right) / a_{0}$.

The stability curve (17) and the approximation (18) are plotted in Fig. 3. The essential features of (17) are a maximum in the range $0<k^{\prime}<a_{0} / \ln \left(a_{0}\right)$, zero growth rate at $k^{\prime}$ $=a_{0} / \ln \left(a_{0}\right)$, and a linear increase in stability with $k^{\prime}$ for large $k^{\prime}$. The long-wavelength cutoff is typical of systems with a
Péclet number, here with the added effect of latent heat embodied in the Stefan number. This demonstrates the competition between the advection and diffusion of heat and momentum (in a harmonic pressure field), the former driving the instability and the latter limiting its extent. The maximum growth rate occurs at approximately

$$
k_{\max }^{\prime} \approx \frac{a_{0}}{-2 \ln \left(a_{0}\right)},
$$

with (nondimensional) growth rate

$$
\sigma_{\max } \approx \frac{a_{0}}{4 S \ln ^{2}\left(a_{0}\right)} .
$$

Translating (19) and (20) back into dimensional quantities, we find that the most unstable mode has angular size given by

$$
\phi_{d e g}=\frac{720^{\circ} \kappa}{u_{0} r_{0}}\left(\frac{r_{0}}{a_{0}}\right) \ln \left(\frac{r_{0}}{a_{0}}\right),
$$

and has growth rate given by

$$
\sigma_{d i m}=\frac{u_{0}}{4 S r_{0} \ln ^{2}\left(r_{0} / a_{0}\right)}\left(\frac{a_{0}}{r_{0}}\right) .
$$

\section{EXTRACTING INFORMATION FROM FIELD OBSERVATIONS}

Field observations of lake stars cannot be controlled. A reasonable estmate for $r_{0}$ is the radius of the wetted (snow) region around the lake stars, and observations [5-7] bound the value as $1.5 \lesssim r_{0} \lesssim 4 \mathrm{~m}$. This is simply because if there were significant excess pressure at this point then the wetting front would have advanced further. However, it is also possible that the effective value of $r_{0}$, say $r_{0}^{e f f}$, is less than this, either because the wetted radius is smaller earlier in the star formation process or because the ambient pressure level is reached at smaller radii. Here, we take $a_{0}$ to be the radius of the roughly circular liquid-filled region at the center of the lake star $\left(r_{\ell}\right)$ as the best approximation during the initial stages of star formation (see Fig. 4). Field observations show that $0.1 \lesssim r_{\ell} \lesssim 0.5 \mathrm{~m},[5-7]$ and hence $0.07 \lesssim r_{\ell} / r_{0} \lesssim 0.15$. We note that Eqs. (21) and (22) are more sensitive to $a_{0} / r_{0}$ than $a_{0}$ or $r_{0}$ independently [12]. With this interpretation of $r_{0}$ we find a reasonable estimate of $u_{0}$ as $1.4 \times 10^{-5} \lesssim u_{0}$ $\lesssim 2.8 \times 10^{-5} \mathrm{~m} / \mathrm{s}$. Using these parameter values, the most unstable mode should have wavelength between $8^{\circ}$ and $130^{\circ}$. Letting the number of branches be $N=360^{\circ} / \phi_{\text {deg }}$, then 3 $<N<45$, and we clearly encompass the observed values for lake stars $(4<N<15)$, but note that values $(N>15)$ are never seen in the field.

Despite the dearth of field observations, many qualitative features embolden our interpretation. For example, the stars with larger values of $a_{0} / r_{0}$ have a larger number of branches. Moreover, for any value of $a_{0} / r_{0}$, our analysis predicts an increase in $N$ with $r_{0}$ and $u_{0}$. Indeed, $u_{0}$ increases with $p_{0}$ (higher water height within the slush layer) and $\Pi$ (less wellpacked snow). Therefore, we ascribe some of the variability 


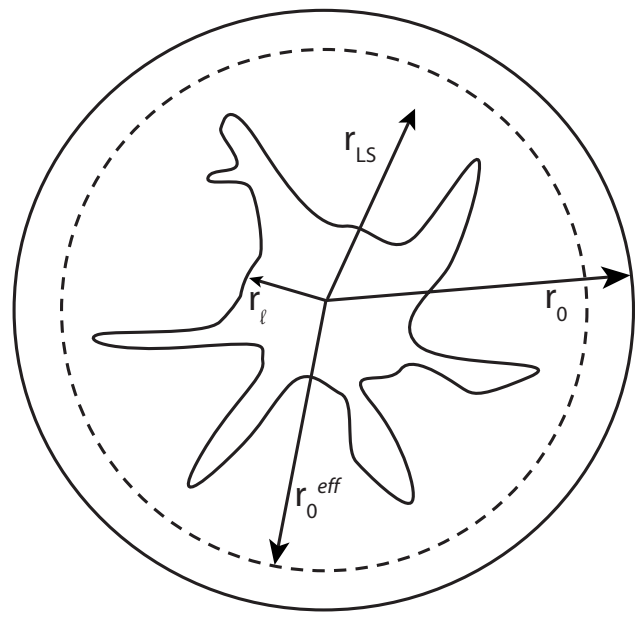

FIG. 4. Schematic showing $r_{0}, r_{0}^{e f f}, r_{L S}$, and $r_{\ell}$.

among field observations to variations in these quantities (which have not been measured in the field) and the remainder to nonlinear effects. Because the dendritic arms are observed long after onset and are far from small perturbations to a radially symmetric pattern, as one might see in the initial stages of the Saffman-Taylor instability, the process involves nonlinear cooperative phenomena. Hence, our model should only approximately agree with observations. Although a rigorous nonlinear analysis of the long-term star evolution process (e.g., [3]) may more closely mirror field observations, the present state of the latter does not warrant that level of detail. Instead, we examine the model physics through a simple proof of concept experiment described presently.

\section{DEMONSTRATING LAKE STARS IN THE LABORATORY}

A 30-cm-diameter circular plate is maintained below freezing $\left(\approx-0.5^{\circ} \mathrm{C}\right)$, and on top of this we place a $0.5-1 \mathrm{~cm}$ deep layer of slush through which we flow $1^{\circ} \mathrm{C}$ water. Given the technical difficulties associated with its production, the grain size, and hence the permeability of the slush layer is not a controlled variable. This fact influences our results quantitatively. In 14 runs we varied the initial size of the water-filled central hole $\left(a_{0}\right)$, that of the circular slush layer $\left(r_{0}\right)$, and the flow rate $(Q)$, which determines $u_{0}$. The flow rate is adjusted manually so that the water level $\left(h_{0}\right)$ in the central hole remains constant [13]. Fingering is observed in every experimental run, and hence we conclude that fingers are a robust feature of the system. Two distinct types of fingering are observed: small-scale fingering (see Fig. 5) that forms early in an experimental run, and larger channel-like fingers (see Fig. 6) that are ubiquitous at later times and often extend from the central hole to the outer edge of the slush. Since the channel-like fingers provide a direct path for water to flow, effectively shorting the Darcy flow within the slush, their subsequent dynamics are not directly analogous to those in natural lake stars. However, in all runs, the initial small-scale fingers have the characteristics of lake stars and hence we focus upon them. We note that, because the larger

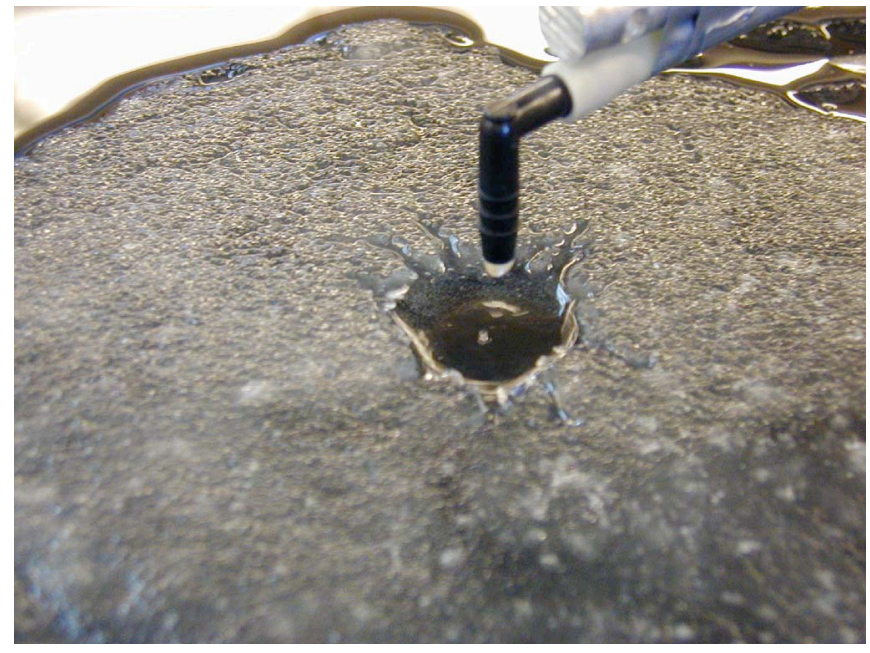

FIG. 5. (Color online) Typical experimental run where smallscale fingers are present. For scale, the nozzle head has diameter of $5 \mathrm{~mm}$.

channel-like fingers emerge out of small-scale fingers, they likely represent the nonlinear growth of the linear modes of instability, a topic left for future study. Finally, we measure the distance between fingers $\left(d_{f}\right)$, so that for each experiment we can calculate $u_{0}=Q /\left(2 \pi r_{0} h_{0}\right), \phi_{\text {calc }} \equiv \phi_{d e g}$, from Eq. (21), and $\phi_{\text {obs }}=180^{\circ} d_{f} /\left(\pi a_{0}\right)$, and we can thereby compare experiment, theory, and field observations.

In Fig. 7 we plot $\phi_{o b s}$ versus $\phi_{\text {calc }}$ for the various field

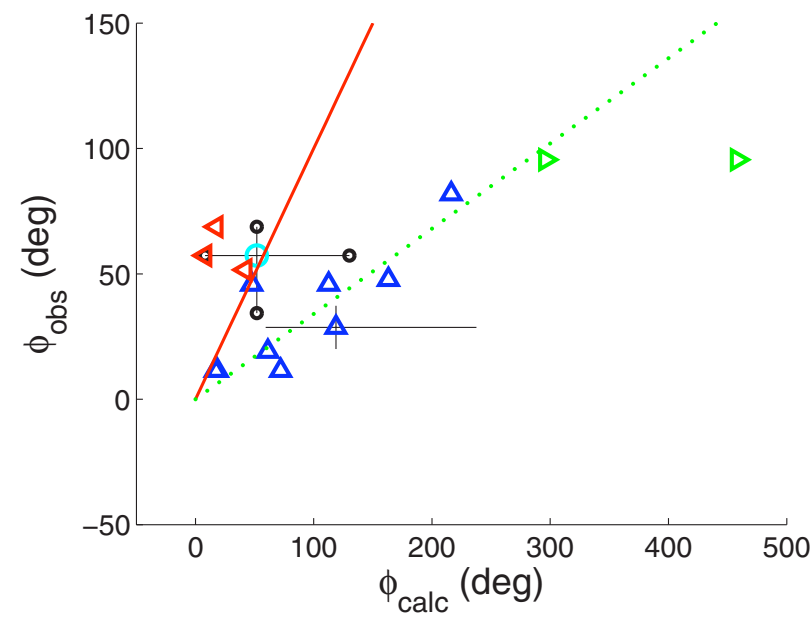

FIG. 7. (Color online) Comparison of theory, experiment, and field observations. Circles are field observations (cyan, best constrained field observation; black, range of plausible field observations), triangles are experimental results (blue upward-pointing triangles were unambiguous; red left-pointing triangles have channels but show no clear small-scale fingers, so channel spacing is taken for $d_{f}$; green right-pointing triangles were compromised by the quality of the images). Errors are approximately $0.3 \mathrm{~cm}, 0.5 \mathrm{~cm}$, $2 \mathrm{~mm}, 5 \mathrm{ml} / \mathrm{min}$, and $0.2 \mathrm{~cm}$ (respectively) for the five measured quantities. All experimental results thus have error bars of at least a factor of 2 in the $x$ coordinate and $30 \%$ in the $y$ coordinate. Typical error bars are shown on one measurement. The solid red line is the theoretical prediction; the dotted green line is the best-fit line to the blue triangles. 


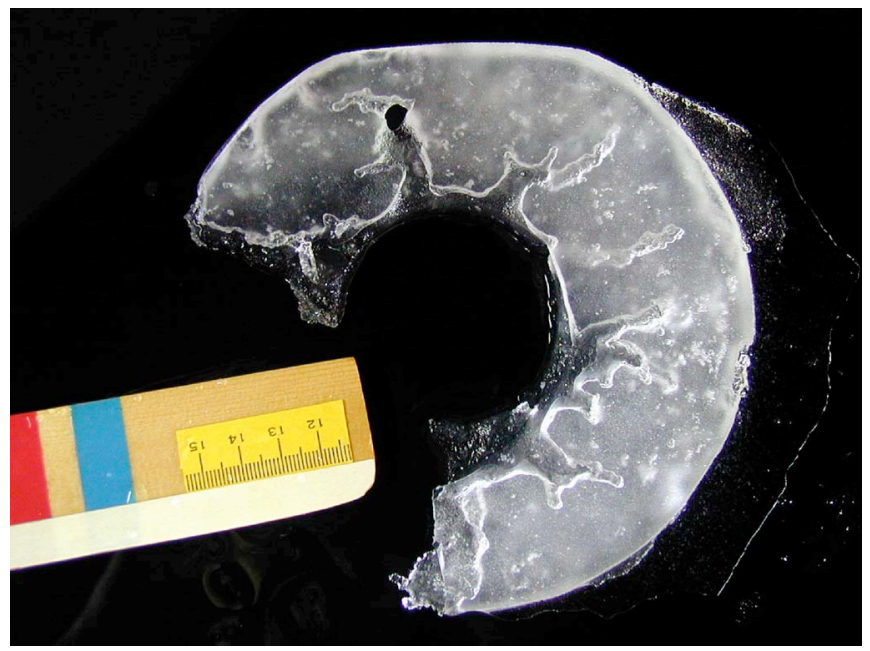

FIG. 6. (Color online) Typical run where channels form. This picture is taken from the underside. Note: part of the slush broke off when it was flipped to image it. The ruler scale is in centimeters.

observations for which we have estimates of parameters, the laboratory experiments described above, and the model [Eq. (21)]. There is a large amount of scatter in both the experimental and observational data, and the data do not lie on the one-to-one curve predicted by the model. However, the experiments are meant to demonstrate the features of the model predictions, and the results have the correct qualitative trend (having a best-fit slope of 0.34). We also attempt to find trends in the experimental data not represented by the model by comparing $y \equiv \phi_{\text {obs }} / \phi_{\text {calc }}$ versus various combinations of control parameters $(\equiv x)$ including $r_{0}, a_{0}, r_{0} / a_{0}, r_{0} u_{0}$, $r_{0} / a_{0} \ln \left(r_{0} / a_{0}\right)$, and $\ln \left(r_{0} / a_{0}\right) /\left(a_{0} u_{0}\right)$. For all plots of $y$ vs $x$, our model predicts a zero slope (and $y$ intercept of 1 ). A nonrandom dependence of $y$ on $x$ would point to failure of some part of our model. Thus, to test the validity of our model, we perform significance tests on all nonflagged data with the null hypothesis being a nonzero slope. In all cases, the null hypothesis is accepted (not rejected) at the 95\% confidence level. Thus, although the agreement is far from perfect, the simple model captures all of the significant trends in the experimental data.

\section{CONCLUSIONS}

By generalizing and quantifying the heuristic ideas of Knight [5], we have constructed a theory that is able to explain the radiating fingerlike patterns on lake ice that we call lake stars. The model yields a prediction for the wavelength of the most unstable mode as a function of various physical parameters that agrees with field observations. Proof of concept experiments revealed the robustness of the fingering pattern, and to leading order the results also agree with the model. There is substantial scatter in the data, and the overall comparison between field observations, model, and experiment demonstrates the need for a comprehensive measurement program and a fully nonlinear theory which will yield better quantitative comparisons. However, the general predictions of our theory capture the leading-order features of the system.

\section{ACKNOWLEDGMENTS}

We thank K. Bradley and J. A. Whitehead for laboratory and facilities support and D. H. Rothman for helpful comments. This research, which began at the Geophysical Fluid Dynamics summer program at the Woods Hole Oceanographic Institution, was partially funded by National Science Foundation (NSF) Grant No. OCE0325296, NSF Grant No. OPP0440841 (J.S.W.), and Department of Energy Grant No. DE-FG02-05ER15741 (J.S.W.). V. C. T. acknowledges financial support from NSF.
[1] T. Y. Hou, J. S. Lowengrub, and M. J. Shelley, J. Comput. Phys. 169, 302 (2001).

[2] M. P. Brenner, L. S. Levitov, and E. O. Budrene, Biophys. J. 74, 1677 (1998); H. Levine and E. Ben-Jacob, Phys. Biol. 1, 14 (2004).

[3] M. C. Cross and P. C. Hohenberg, Rev. Mod. Phys. 65, 851 (1993); I. S. Aranson and L. S. Tsimring, ibid. 78, 641 (2006).

[4] N. Goldenfeld, P. Y. Chan, and J. Veysey, Phys. Rev. Lett. 96, 254501 (2006); M. B. Short, J. C. Baygents, and R. E. Goldstein, Phys. Fluids 18, 083101 (2006).

[5] C. A. Knight, in Structure and Dynamics of Partially Solidified Systems, edited by D. E. Loper (Martinus Nijhoff, Dordrecht, 1987), pp. 453-465.

[6] K. B. Katsaros, Bull. Am. Meteorol. Soc. 64, 277 (1983).

[7] A. H. Woodcock, Limnol. Oceanogr. 10, R290 (1965).

[8] M. G. Worster, Annu. Rev. Fluid Mech. 29, 91 (1997).

[9] S. Fitzgerald and A. W. Woods, Nature (London) 367, 450
(1994).

[10] N. Schorghofer, B. Jensen, A. Kudrolli, and D. H. Rothman, J. Fluid Mech. 503, 357 (2004).

[11] A finite body of fresh water cooled from above will have a maximum below ice temperature of $4{ }^{\circ} \mathrm{C}$.

[12] For the later stages of growth, clearly in the nonlinear regime not treated presently, $a_{0}$ may also be interpreted as the radius of the lake star $\left(r_{L S}\right)$. Field observations show that $1 \lesssim r_{L S}$ $\$ 2 \mathrm{~m}[5-7]$ and hence $0.3 \lessgtr r_{L S} / r_{0} \lesssim 0.6$.

[13] In many of the runs, we begin the experiment without the central hole. In practice, however, the first few drops of warm water create a circular hole with radius one to three times the radius of the water nozzle $\left(0.5<a_{0}<1.0 \mathrm{~cm}\right)$. It is significantly more difficult to prepare a uniform permeability sample with a circular hole initially present; these runs are therefore more difficult to interpret. 